\title{
Dust reddening in star-forming galaxies
}

\section{Ting $\mathrm{Xiao}^{1} \dagger$, Tinggui Wang ${ }^{2}$, Huiyuan Wang ${ }^{2}$, Hongyan Zhou ${ }^{2,3}$, Honglin $\mathrm{Lu}^{2}$ and Xiaobo Dong ${ }^{2}$}

\author{
${ }^{1}$ Partner Group of the Max Planck Institute for Astrophysics and Key Laboratory for \\ Research in Galaxies and Cosmology of Chinese Academy of Sciences, Shanghai Astronomical \\ Observatory, Nandan Road 80, Shanghai 200030, China \\ ${ }^{2}$ Key Laboratory for Research in Galaxies and Cosmology, Department of Astronomy, \\ University of Science \& Technology of China, Hefei, Anhui 230026, China \\ ${ }^{3}$ Polar Research Institute of China, Jinqiao Road 451, Shanghai 200136, China
}

\begin{abstract}
Dust is a crucial component of galaxies in modifying the observed properties of galaxies. Previous studies have suggested that dust reddening in star-forming galaxies is correlated with star formation rate (SFR), luminosity, gas-phase metallicity $(Z)$, stellar mass $\left(\mathrm{M}_{*}\right)$ and inclination. In this work we investigate the fundamental relations between dust reddening and physical properties of galaxies, and obtain a well-defined empirical recipe for dust reddening. The empirical formulae can be incorporated into semi-analytical models of galaxy formation and evolution to estimate the dust reddening and facilitate comparison with observations.
\end{abstract}

Keywords. galaxies: ISM — galaxies: abundances - HII regions — dust, extinction

We select a large sample of $\sim 22000$ well-defined star-forming disc galaxies from the Sloan Digital Sky Survey (SDSS), and measure galaxy properties including $\mathrm{H} \alpha$ luminosity, $\mathrm{H} \alpha$ surface brightness $B_{\mathrm{H} \alpha}, \mathrm{M}_{*}, Z$ and axial ratio $b / a$ (Xiao et al. 2012). The luminosity and surface brightness have been corrected for aperture and dust extinction. As an examination, when we compare the SFRs estimated from corrected $\mathrm{H} \alpha$ luminosity with that estimated from far infrared luminosity, the scatter is as small as 0.13 dex. We divide the sample into different metallicity bins, and find the best-fit empirical relations. ${ }^{1}$ The scatter of $E(\mathrm{~B}-\mathrm{V})$ residual is $0.068 \mathrm{mag}$, which can be explained by considering the measurement errors and calibration uncertainties in physical parameters like metallicity.

We construct a simple toy model of dust extinction for a disc galaxy to compare with the observations. In the model, we adopt several assumptions: (1) The diffuse interstellar medium is smoothly distributed and follows exponential laws in the vertical and radial directions; (2) dust is coupled with gas; (3) star formation follows the Kennicutt-Schmidt law (Kennicutt 1998). From the comparison, we find the observed trends between dust reddening and $\mathrm{H} \alpha$ surface brightness could be reproduced by a plane-parallel toy model. In the model that best reproduces the observational trends, dust-to-gas ratio is assumed to be proportional to $Z^{0.7}$, not the linear relation widely adopted.

\section{References}

Kennicutt, R. C., Jr. 1998, ApJ, 498, 541

Xiao, T., Wang, T.-G., Wang, H.-Y., Zhou, H.-Y., Lu, H.-L., \& Dong, X.-B. 2012, MNRAS, 421, 486

$\dagger$ LAMOST fellow; Email: xiaoting@shao.ac.cn

1. $E(\mathrm{~B}-\mathrm{V})=(0.27 \pm 0.02)\left(\frac{Z}{Z_{\odot}}\right)^{0.71 \pm 0.01} B_{\mathrm{H} \alpha, 40}^{0.23}(b / a)^{-0.4}$ 